\title{
Noninvasive Evaluation of Myocardial Systolic Dysfunction in the Early Stage of Kawasaki Disease:A Speckle-Tracking Echocardiography Study
}

\author{
Mohammad-Nasir Hematian ${ }^{1}$; Shirin Torabi ${ }^{2, *}$; Elaheh MalaKan-Rad ${ }^{1,3}$; Keyhan Sayadpour- \\ Zanjani $^{1,3}$; Vahid Ziaee $^{1,4}$; Masoumeh Lotfi-Tolkaldany ${ }^{3}$ \\ ${ }^{1}$ Children's Medical Center, Pediatric Center of Excellence, Tehran, IR Iran \\ ${ }^{2}$ Gonabad University of Medical Sciences, Gonabad, IR Iran \\ 3 Department of Pediatrics, Tehran University of Medical Sciences, Tehran, IR Iran \\ 4 Rheumatology Research Center, Tehran University of Medical Sciences, Tehran, IR Iran \\ ${ }_{5}^{5}$ Repartment of Basic and Clinical Research, Tehran Heart Center, Tehran University of Medical Sciences, Tehran, IR Iran \\ *Corresponding author: Shirin Torabi, Gonabad University of Medical Sciences, P. O. Box: 9691844177, Gonabad, IR Iran. Tel:+98-9153025709, Fax:+98-53572-3815, E-mail: sh.torabi58@yahoo.com
}

Received: June 11, 2014; Accepted: December 12, 2014

Background: Evaluation of myocardial function by speckle-tracking echocardiography is a new method for the early diagnosis of systolic dysfunction.

Objectives: We aimed to determine myocardial speckle-tracking echocardiography indices in Kawasaki Disease (KD) patients and compare them with the same indices in control subjects.

Patients and Methods: Thirty-two patients (65.5\% males) with KD and 19 control subjects with normal echocardiography participated in this study. After their demographic characteristics and clinical findings were recorded, all the participants underwent transthoracic echocardiography. Strain(S),Strain Rate(SR), Time to PeakStrain(TPS), and Strain Rate(TPSR), longitudinal velocity and view point velocity images in the two, three, and four-chamber views were semi-automatically obtained via speckle-tracking echocardiography.

Results: Among the patients, Twenty-four cases (75\%) were younger than 4 years. Mean global S and SR was significantly reduced in the KD patients compared to controls (17.03 \pm 1.28 vs. $20.22 \pm 2.14 \%$ and $1.66 \pm 0.16$ vs. $1.97 \pm 0.251$ /second, respectively), while there were no significant differences regarding mean TPS, TPSR, longitudinal velocity and view point velocity. Using repeated measure of analysis of variances, we observed that $S$ and SR decreased from base to apical level in both groups. The change in the pattern of age adjusted mean $S$ and SR across levels was significantly different between the groups ( $\mathrm{P}<0.001$ for both parameters).

Conclusions:We showed changes in Sand SR assessed in KD patients versus control subjects in the acute phase of KD. However, we suggest that further studies be undertaken to compare S and SR in the acute phase and thereafter in KD patients.

Keywords: Mucocutaneous Lymph Node Syndrome; Ventricular Function, Left; Myocardial Velocity; Strain Rate Imaging; Myocardial Systolic Dysfunction; Speckle-Tracking Echocardiography

\section{Background}

The principal cause of myocardial infarction in children is Kawasaki Disease (KD) (1). In KD patients, myocardial dysfunction due to coronary disorders is the most important complication during long-term follow-up (1).

Several studies have been undertaken to find a noninvasive method for the identification of systolic myocardial dysfunction in KD patients at the early stage of coronary insufficiency (2-5). Echocardiography has been employed as a noninvasive method in some studies for early diagnosis (1). Speckle-tracking echocardiography is a new method in echocardiographic imaging and is suggested for the evaluation of systolic myocardial dysfunction analysis. Speckle-tracking-derived parameters are considered as valid indices for the detection of sub-clinical myocardial systolic dysfunction secondary to coronary insufficiency (6).

A few studies conducted on longitudinal and circum- ferential markers have introduced myocardial Strain (S) and Strain Rate (SR) as the early markers of myocardial dysfunction in patients suffering from $\operatorname{KD}(1,7)$. While some of these studies reported a reduction in S and SR in KD patients, others reported no difference in SR between control groups and KD patients $(1,7,8)$. Note that all of these studies investigated only S and SR as the markers of systolic dysfunction in KD patients $(1,7,9)$.

\section{Objectives}

Therefore, we sought to assess changes in myocardial time to peak, View Point (VP) velocity, longitudinal velocity, global S, and SR, in addition to S and SR, as a noninvasive method for the early diagnosis of the effect of KD on coronary artery insufficiency during the acute phase in KD patients.

Copyright (C) 2015, Growth \& Development Research Center. This is an open-access article distributed under the terms of the Creative Commons Attribution-NonCommercial 4.0 International License (http://creativecommons.org/licenses/by-nc/4.0/) which permits copy and redistribute the material just in noncommercial usages, provided the original work is properly cited. 


\section{Patients and Methods}

\subsection{Study Population}

Thirty-two patients diagnosed with KD and 19 control subjects were recruited in this study. Participants with a history of hypertension, diabetes, coronary artery disease, myocardial infarction, stroke, congenital heart disease, acquired valvular disease, dysrhythmia, or congestive heart failure were excluded. Patients in the acute phase of the disease were enrolled before the administration of intravenous immunoglobulin.

Demographic characteristics and clinical findings, including systemic blood pressure (systolic and diastolic) and body weight, were recorded in a specially designed questionnaire. Thereafter, all the participants underwent a transthoracic echocardiographic examination. Written informed consent was obtained from the parents of all the participants after they had been provided with a thorough explanation about the purpose of the study.

The control subjects were selected from healthy children who were referred to the Cardiovascular Clinic for innocent murmur and had normal echocardiography.

\subsection{Echocardiographic Study}

Echocardiographic evaluations were performed by the same operator, using a MyLab60 echo machine (Esaote, S.P.A Mylab60, Florence, Italy, Class 2A). Echocardiography was done with a multi-frequency phased array 122 (3 - $8 \mathrm{MHZ)} \mathrm{transducer} \mathrm{for} \mathrm{patients} \mathrm{under} 1$ year old and a phased array 230 (1.6 - 4 MHz) transducer for patients older than 1 year. Video clips of the two and three-chamber views were recorded as two-dimensional harmonic image cine-loops. Echocardiographic view was done in a frame rate between 40 - 75 fps under good quality ECG signal. These records were analyzed by XStrain ${ }^{\mathrm{TM}}$ software license (Esaote, Florence, Italy, Class 2A) and stored on the computer for off-line analysis. Based on the algorithm of optical flow analysis, XStrain ${ }^{\mathrm{TM}}$ software was utilized to calculate longitudinal myocardial VP velocity, longitudinal velocity, S, SR, Time to Peak Systolic strain (TPS), and Time to Peak Strain Rate (TPSR) from the digitized twodimensional video clips.

Initially, the frame at the beginning of systole, when the endocardial border is better visualized, was chosen. After the manual determination of the 3 main points on the mitral annulus and the apex of the endocardial border of the left ventricle in the first patient, the Aided Heart Segmentation (AHS) tool was employed for the rest of the patients to identify the best border. In other words, in the first patient, each point had to be selected and manually placed, whereas in the other patients, the AHS tool suggested which myocardial points (landmarks) should be tracked $(1,6)$. The frame of the AHS mode was drawn based upon maximum likelihood neighboring points. After the semi-automatic identification of the points by the AHS mode, the points were checked by a skilled operator and the inaccurate points were corrected for the confirmation of the best border. Longitudinal velocity, VP velocity, S, and SR were calculated for each point.

Thirteen equidistant points were tracked in the apical four, two, and three-chamber views (Figure 1) to draw 1 subdivision in 6 equidistant segments (each including apical, mid, and basal segments), allowing the use of an 18-segment model. The points positioned in a semi-automatic way (AHS mode) were selected from the septal side of the mitral annulus in the apical four-chamber view (Figure 1), from the inferior wall of the mitral annulus in the apical two-chamber view, and from the points starting from the posterior wall to the anteroseptal wall in the three-chamber view. Once the procedure was completed, a view point represented by a triangle was shown in the selected cycle. VP velocity was calculated based on the movement of the wall edge from this point. Longitudinal velocity, VP velocity, TPS, TPSR, S, and SR images were semi-automatically obtained using XStrain ${ }^{\mathrm{TM}}$ software. For intra-observer variability, all the variables for all the segments were measured in 8 subjects by one skilled operator at two-week intervals.

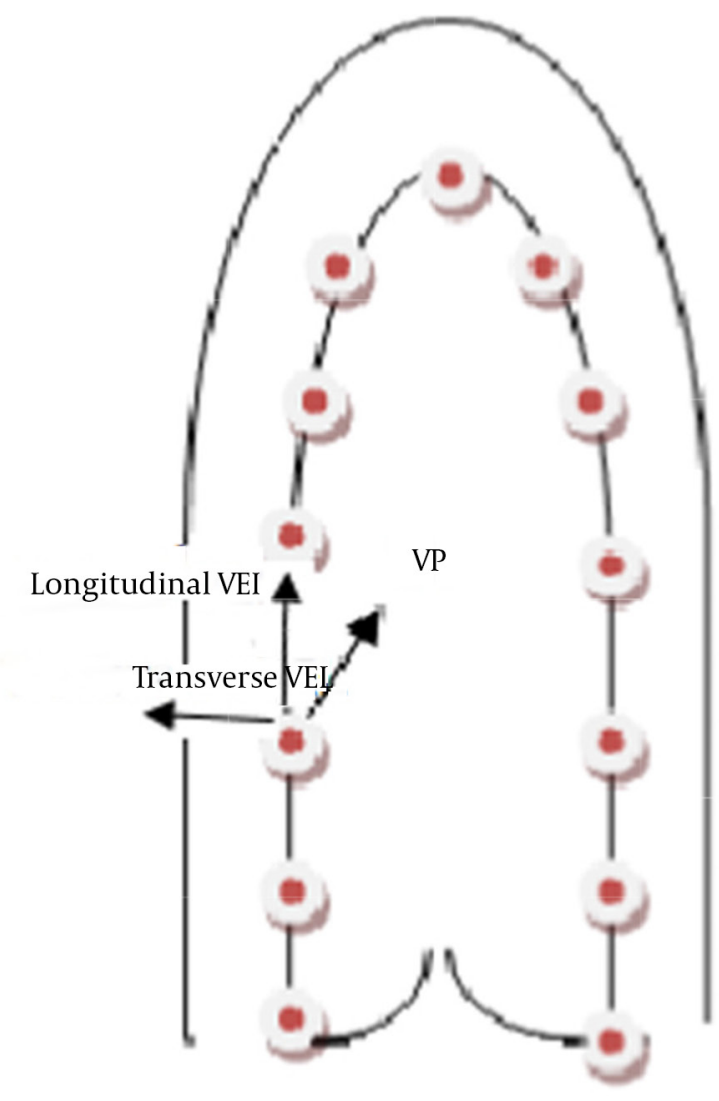

Figure 1. Thirteen Equidistant Points Were Tracked in Longitudinal Axi to Determine View Point Velocity and Longitudinal Velocity 
Hematian $M$ et al.

\subsection{Statistical Analysis}

Mean and Standard Deviation were reported for all the quantitative echocardiographic data, and the Pearson correlation test was used to test intra-observer variability. Subsequently, precision was reported with the Pearson correlation coefficient ( $\mathrm{r}$ ) and with $\mathrm{C}$ bias, which is a bias correction factor for accuracy measurement. The Mann-Whitney U test was utilized to compare the differences between the groups. Repeated measure of analysis of variances was applied to compare the changes of S, SR, TPS and TPSR across different levels of left ventricular myocardium (basal, mid and apical) between the KD patients and controls. Comparisons were done while the variables were adjusted for age; because of the importance of the effect of age in the changes of statistical significance, it was set at a $P$ value smaller than 0.05 .

\section{Results}

\subsection{Patient Characteristics}

Of a total of $35 \mathrm{KD}$ patients examined by echocardiography, 32 patients had complete echocardiographic data, and of 21 control subjects undergoing echocardiographic examination, 2 were eliminated from our study due low quality imaging.

Table 1 demonstrates the demographic characteristics of the study groups. Most of the KD patients were male (21; 65.5\%), and there were no significant differences between the two groups regarding gender and age (Table 1). The mean systolic and diastolic blood pressures and ejection fraction were not different between the groups, and nor was there any significant difference between the two groups in terms of body weight $(\mathrm{P}=0.40)$.

\subsection{Intra-Observer Variability}

Intra-observer variability for $C$ bias was $r=0.90(95 \%$ confidence interval: $0.87-0.93 ; \mathrm{P}<0.01)$, and the bias correction factor (accuracy) was 0.94 .

\subsection{Myocardial Systolic Markers Analysis}

\subsubsection{Four-Chamber View}

In the four-chamber view, there were significant differences in $S$ between KD patients and control group in the basal lateral, mid-wall lateral, basal septal, and mid-wall septal views, as can be seen in Table 2. The SR in the basal lateral, basal septal, apical septal, and mid-wall septal views was lower in the KD patients than in the control group (1.75 \pm 0.46 vs. $2.21 \pm 0.56,1.73 \pm 0.47$ vs. $2.09 \pm 0.54$, $1.53 \pm 0.29$ vs. $1.86 \pm 0.43$, and $1.48 \pm 0.34$ vs. $1.72 \pm 0.26$, respectively). The details are given in Table 2 . The apical lateral and apical septal VP velocity markers were significantly higher in the KD patients, while there was no significant difference between the KD patients and the control subjects in long velocity indices.

\subsubsection{Two-ChamberView}

Compared to the controls, the KD patients had a significantly lower mean S and SR in the basal and mid-wall anterior and basal inferior (Table 3 ).

\subsubsection{Three-Chamber View}

The mean S and SR of the KD patients were significantly reduced in the basal and mid-wall anteroseptal and the basal, mid-wall, and apical posterior in the three-chamber view. Table 4 presents the details. No significant differences were seen between the two groups in VP velocity and longitudinal velocity markers.

\begin{tabular}{|c|c|c|c|}
\hline Variables & Control, $n=19$ & KD Patients, $n=32$ & P Value \\
\hline Mean age, $y$ & & & 0.19 \\
\hline$<4$ & $15(79)$ & $24(75)$ & \\
\hline $5-8$ & $1(5)$ & $5(16)$ & \\
\hline$\geq 9$ & $3(16)$ & $3(9)$ & \\
\hline Male gender & $16(84)$ & $21(65.5)$ & 0.17 \\
\hline Weight, kg & $16.45 \pm 11.51$ & $16.03 \pm 10.03$ & 0.40 \\
\hline Heart rate, beat/min & $110 \pm 11$ & 124.00 & 0.18 \\
\hline Ejection fraction, \% & $64.81 \pm 4.19$ & $63.09 \pm 4.30$ & 0.13 \\
\hline Systolic blood pressure, mm Hg & $93.46 \pm 6.94$ & $92.60 \pm 5.95$ & 0.15 \\
\hline Diastolic blood pressure, $\mathrm{mm} \mathrm{Hg}$ & $49.61 \pm 6.94$ & $50.12 \pm 6.54$ & 0.17 \\
\hline
\end{tabular}

\footnotetext{
a Abbreviation: KD, Kawasaki Disease.

b Data are presented No (\%) or mean SD.
} 
Hematian M et al.

\begin{tabular}{|c|c|c|c|c|c|c|}
\hline \multirow[t]{2}{*}{ Variables } & \multicolumn{3}{|c|}{ Lateral Wall } & \multicolumn{3}{|c|}{ Septal Wall } \\
\hline & Control $^{\mathrm{b}}$ & KD Patients $^{\mathrm{C}}$ & P Value & Control $^{\mathrm{b}}$ & KD Patients ${ }^{\mathrm{C}}$ & P Value \\
\hline \multicolumn{7}{|l|}{ Strain, \% } \\
\hline Basal & $23.03 \pm 4.07$ & $17.52 \pm 3.65$ & 0.01 & $21.45 \pm 5.38$ & $17.62 \pm 3.78$ & 0.01 \\
\hline Mid-wall & $19.41 \pm 3.31$ & $15.38 \pm 2.81$ & $<0.001$ & $20.17 \pm 3.40$ & $17.88 \pm 3.55$ & 0.03 \\
\hline Apical & $16.33 \pm 4.13$ & $16.66 \pm 2.73$ & 0.45 & $15.71 \pm 3.84$ & $13.83 \pm 4.40$ & 0.19 \\
\hline \multicolumn{7}{|c|}{ Strain rate $(\mathbf{1} / \mathbf{s})$} \\
\hline Basal & $2.21 \pm .56$ & $1.75 \pm .46$ & 0.01 & $2.09 \pm .54$ & $1.73 \pm .47$ & 0.03 \\
\hline Mid-wall & $1.66 \pm .43$ & $1.59 \pm .42$ & 0.54 & $1.86 \pm .43$ & $1.53 \pm .29$ & 0.01 \\
\hline Apical & $1.60 \pm 0.31$ & $6.32 \pm 27.31$ & 0.32 & $1.72 \pm .26$ & $1.48 \pm .34$ & 0.01 \\
\hline \multicolumn{7}{|c|}{ VP velocity, ms } \\
\hline Basal & $141.56 \pm 43.51$ & $139.93 \pm 39.19$ & 0.96 & $139.25 \pm 34.90$ & $164.21 \pm 60.39$ & 0.25 \\
\hline Mid-wall & $154.50 \pm 112.65$ & $207.50 \pm 88.54$ & 0.005 & $184.56 \pm 95.32$ & $265.28 \pm 146.75$ & 0.13 \\
\hline Apical & $281.37 \pm 119.45$ & $243.59 \pm 117.70$ & 0.23 & $197.25 \pm 102.86$ & $280.40 \pm 137.45$ & 0.04 \\
\hline \multicolumn{7}{|c|}{ VP velocity, $\mathrm{cm} / \mathrm{s}$} \\
\hline Basal & $3.92 \pm 1.13$ & $3.92 \pm 1.35$ & 0.75 & $3.81 \pm 0.98$ & $3.82 \pm 1.05$ & 0.71 \\
\hline Mid-wall & $1.81 \pm .70$ & $1.70 \pm .89$ & 0.34 & $1.79 \pm 0.65$ & $1.77 \pm 0.70$ & 0.45 \\
\hline Apical & $0.89 \pm 0.54$ & $0.70 \pm 0.37$ & 0.35 & $0.97 \pm 0.60$ & $1.02 \pm 0.49$ & 0.48 \\
\hline \multicolumn{7}{|c|}{ Long velocity, ms } \\
\hline Basal & $131.56 \pm 52.69$ & $175.50 \pm 93.06$ & 0.12 & $150.12 \pm 56.67$ & $157.75 \pm 72.00$ & 0.95 \\
\hline Mid-wall & $136.93 \pm 57.57$ & $168.40 \pm 103.15$ & 0.35 & $155.18 \pm 42.55$ & $155.80 \pm 69.04$ & 0.91 \\
\hline Apical & $190.81 \pm 116.84$ & $191.37 \pm 132.49$ & 0.84 & $193.87 \pm 111.66$ & $202.71 \pm 116.42$ & 0.80 \\
\hline \multicolumn{7}{|c|}{ Long velocity, $\mathrm{cm} / \mathrm{s}$} \\
\hline Basal & $4.25 \pm 1.27$ & $4.69 \pm 2.16$ & 0.71 & $4.26 \pm 0.93$ & $4.69 \pm 0.93$ & 0.16 \\
\hline Mid-wall & $2.93 \pm 1.09$ & $2.93 \pm 1.53$ & 0.39 & $2.38 \pm 0.65$ & $2.63 \pm 0.71$ & 0.35 \\
\hline Apical & $1.35 \pm 0.70$ & $1.43 \pm 10.28$ & 0.46 & $0.63 \pm 0.29$ & $0.83 \pm 0.39$ & 0.07 \\
\hline
\end{tabular}

a Abbreviations: KD, Kawasaki Disease, VP, View Point.

b $(n=19)$.

c $(\mathrm{n}=32)$.

\begin{tabular}{|c|c|c|c|c|c|c|}
\hline \multirow[t]{2}{*}{ Variables } & \multicolumn{3}{|c|}{ Anterior Wall } & \multicolumn{3}{|c|}{ Inferior Wall } \\
\hline & Control $^{\mathrm{b}}$ & KD Patients ${ }^{\mathrm{C}}$ & P Value & Control $^{\mathrm{b}}$ & KD Patients ${ }^{\mathrm{C}}$ & P Value \\
\hline \multicolumn{7}{|l|}{ Strain, \% } \\
\hline Basal & $22.19 \pm 6.37$ & $18.41 \pm 5.37$ & 0.03 & $23.92 \pm 5.05$ & $20.13 \pm 4.43$ & 0.009 \\
\hline Mid-wall & $20.71 \pm 4.91$ & $16.59 \pm 4.72$ & 0.01 & $22.11 \pm 4.86$ & $20.55 \pm 4.68$ & 0.31 \\
\hline Apical & $16.59 \pm 2.97$ & $17.35 \pm 3.09$ & 0.48 & $15.97 \pm 3.03$ & $14.67 \pm 3.76$ & 0.11 \\
\hline \multicolumn{7}{|c|}{ Strain rate $(\mathbf{1} / \mathbf{s})$} \\
\hline Basal & $2.32 \pm 0.51$ & $1.77 \pm 0.42$ & 0.001 & $2.47 \pm 0.40$ & $1.93 \pm 0.45$ & $<0.001$ \\
\hline Mid-wall & $1.94 \pm 0.39$ & $1.74 \pm 0.51$ & 0.05 & $2.06 \pm 0.53$ & $1.77 \pm 0.44$ & 0.12 \\
\hline Apical & $1.57 \pm 0.32$ & $1.58 \pm 0.31$ & 0.76 & $1.47 \pm 0.29$ & $1.53 \pm 0.39$ & 0.67 \\
\hline \multicolumn{7}{|c|}{ VP velocity, ms } \\
\hline Basal & $142.50 \pm 59.89$ & $154.78 \pm 76.83$ & 0.93 & $128.18 \pm 38.73$ & $140.87 \pm 46.79$ & 0.62 \\
\hline Mid-wall & $228.06 \pm 150.38$ & $264.90 \pm 177.13$ & 0.69 & $145.68 \pm 103.93$ & $209.78 \pm 105.61$ & 0.02 \\
\hline Apical & $207.00 \pm 107.81$ & $259.25 \pm 115.18$ & 0.11 & $247.56 \pm 155.62$ & $204.90 \pm 93.58$ & 0.92 \\
\hline \multicolumn{7}{|c|}{ VP velocity, $\mathrm{cm} / \mathrm{s}$} \\
\hline Basal & $3.57 \pm 0.88$ & $3.65 \pm 1.30$ & 0.88 & $4.48 \pm 0.82$ & $4.67 \pm 1.10$ & 0.71 \\
\hline Mid-wall & $1.69 \pm 0.45$ & $1.42 \pm 0.92$ & 0.03 & $1.71 \pm 0.59$ & $2.04 \pm 0.74$ & 0.09 \\
\hline Apical & $0.86 \pm 0.59$ & $0.87 \pm 0.41$ & 0.41 & $0.90 \pm 0.46$ & $0.87 \pm 0.44$ & 0.99 \\
\hline \multicolumn{7}{|c|}{ Longitudinal velocity, ms } \\
\hline Basal & $113.62 \pm 55.50$ & $154.87 \pm 78.75$ & 0.04 & $140.68 \pm 29.47$ & $145.84 \pm 47.12$ & 0.95 \\
\hline Mid-wall & $106.81 \pm 35.43$ & $142.96 \pm 59.24$ & 0.06 & $144.25 \pm 45.50$ & $154.15 \pm 55.79$ & 0.69 \\
\hline Apical & $156.68 \pm 123.02$ & $144.37 \pm 123.82$ & 0.61 & $161.81 \pm 69.26$ & $208.65 \pm 80.17$ & 0.06 \\
\hline \multicolumn{7}{|c|}{ Longitudinal velocity, $\mathrm{cm} / \mathrm{s}$} \\
\hline Basal & $4.13 \pm 1.29$ & $4.29 \pm 1.52$ & 0.69 & $5.05 \pm 1.17$ & $5.56 \pm 2.70$ & 0.69 \\
\hline Mid-wall & $2.51 \pm 1.01$ & $2.66 \pm 1.22$ & 0.54 & $2.85 \pm 0.69$ & $3.36 \pm 2.11$ & 0.60 \\
\hline Apical & $1.21 \pm 0.57$ & $1.21 \pm 0.91$ & 0.38 & $0.71 \pm 0.23$ & $1.29 \pm 1.10$ & 0.005 \\
\hline
\end{tabular}

a Abbreviations: KD, Kawasaki Disease, VP, View Point.

$\mathrm{b}(\mathrm{n}=19)$.

c $(\mathrm{n}=32)$. 
Table 4. Comparison of Left Ventricular Peak Systolic Indices Between the KD Patients and the Control Subjects in the Three-Chamber View $^{\mathrm{a}}$

\begin{tabular}{|c|c|c|c|c|c|c|}
\hline \multirow[t]{2}{*}{ Variables } & \multicolumn{3}{|c|}{ Anteroseptal Wall } & \multicolumn{3}{|c|}{ Posterior Wall } \\
\hline & Control $^{b}$ & KD Patients $^{\mathrm{C}}$ & PValue & Control $^{b}$ & KD Patients ${ }^{c}$ & P Value \\
\hline \multicolumn{7}{|l|}{ Strain, \% } \\
\hline Basal & $23.68 \pm 6.26$ & $18.77 \pm 5.32$ & 0.004 & $24.29 \pm 5.15$ & $17.38 \pm 4.98$ & $<0.001$ \\
\hline Mid-wall & $22.58 \pm 2.97$ & $16.84 \pm 3.63$ & $<0.001$ & $20.51 \pm 4.32$ & $15.51 \pm 4.15$ & $<0.001$ \\
\hline Apical & $17.34 \pm 3.52$ & $16.15 \pm 2.98$ & 0.24 & $17.89 \pm 1.92$ & $15.27 \pm 3.97$ & 0.002 \\
\hline \multicolumn{7}{|c|}{ Strain rate (1/s) } \\
\hline Basal & $2.61 \pm 0.54$ & $1.87 \pm 0.60$ & $<0.001$ & $2.55 \pm 0.67$ & $1.90 \pm 0.68$ & 0.003 \\
\hline Mid-wall & $2.11 \pm 0.50$ & $1.65 \pm 0.41$ & 0.004 & $2.18 \pm 0.48$ & $1.59 \pm 0.52$ & 0.001 \\
\hline Apical & $1.58 \pm 0.30$ & $1.40 \pm 0.30$ & 0.06 & $1.52 \pm 0.30$ & $1.55 \pm 0.35$ & 0.88 \\
\hline \multicolumn{7}{|c|}{ VP velocity, ms } \\
\hline Basal & $118.81 \pm 40.73$ & $173.18 \pm 142.87$ & 0.12 & $133.81 \pm 51.84$ & $166.06 \pm 115.12$ & 0.12 \\
\hline Mid-wall & $222.43 \pm 143.71$ & $218.25 \pm 135.19$ & 0.93 & $211.62 \pm 128.60$ & $213.96 \pm 204.23$ & 0.83 \\
\hline Apical & $222.62 \pm 124.99$ & $236.09 \pm 175.88$ & 0.71 & $326.62 \pm 133.07$ & $280.86 \pm 150.32$ & 0.22 \\
\hline \multicolumn{7}{|c|}{ VP velocity, $\mathrm{cm} / \mathrm{s}$} \\
\hline Basal & $3.42 \pm 1.20$ & $3.48 \pm 0.79$ & 0.31 & $3.64 \pm 0.96$ & $4.24 \pm 1.15$ & 0.07 \\
\hline Mid-wall & $1.60 \pm 0.56$ & $1.41 \pm 0.64$ & 0.12 & $1.61 \pm 0.58$ & $2.08 \pm 0.87$ & 0.10 \\
\hline Apical & $0.90 \pm 0.45$ & $1.18 \pm 0.96$ & 0.44 & $1.03 \pm 0.33$ & $1.34 \pm 0.95$ & 0.79 \\
\hline \multicolumn{7}{|c|}{ Long velocity, ms } \\
\hline Basal & $124.93 \pm 49.29$ & $153.90 \pm 111.29$ & 0.33 & $141.25 \pm 115.61$ & $161.50 \pm 140.21$ & 0.25 \\
\hline Mid-wall & $128.31 \pm 61.26$ & $168.40 \pm 141.73$ & 0.48 & $116.56 \pm 58.81$ & $146.61 \pm 117.13$ & 0.54 \\
\hline Apical & $152.06 \pm 109.88$ & $166.0 \pm 119.76$ & 0.63 & $147.43 \pm 77.83$ & $150.78 \pm 179.99$ & 0.19 \\
\hline \multicolumn{7}{|c|}{ Long velocity, $\mathrm{cm} / \mathrm{s}$} \\
\hline Basal & $4.28 \pm 1.14$ & $4.85 \pm 1.23$ & 0.08 & $4.59 \pm 1.76$ & $4.88 \pm 1.59$ & 0.64 \\
\hline Mid-wall & $2.78 \pm 0.75$ & $3.02 \pm 0.97$ & 0.45 & $2.74 \pm 0.97$ & $3.20 \pm 1.07$ & 0.13 \\
\hline Apical & $1.36 \pm 0.65$ & $1.76 \pm 0.99$ & 0.17 & $1.04 \pm 0.45$ & $1.23 \pm 0.49$ & 0.17 \\
\hline
\end{tabular}

\subsubsection{Global Indices}

In overall, in univariate analysis mean global S and SR was significantly reduced in the KD patients $(17.03 \pm 1.28$ vs. $20.22 \pm 2.14 \%$ and $1.66 \pm 0.16$ vs. $1.97 \pm 0.251 /$ second, respectively) (Table 5), while there was no significant difference between the KD patients and the control subjects in global mean time to peak systolic indices. Using repeated measure of analysis of variances, we observed that $S$ and SR decreased from base to apical level in both groups (Figure 2). The change in the pattern of age adjusted mean $S$ and SR across levels was different between the groups ( $\mathrm{P}<0.001$ for both parameters).
As it is shown in Figure 2 ( $A$ and $B$ ) reduction of $S$ and SR was sharply in the controls, while this The change in age adjusted mean time to peak strain from basal to apical level in KD patients was also significantly different from that in controls $(\mathrm{P}=0.001)$ (Figure $2 \mathrm{C}$ ), showing that in controls mean time to peak strain decreased from base to apical level, whereas it remained unchanged across the levels in KD patients. Mean time to peak systolic strain rate, adjusted for age, has almost similar change across levels in both groups $(\mathrm{P}=0.060)$ (Figure $2 \mathrm{D}$ ). 
Table 5. Results of Multivariate Analysis Comparing Global Values of Echocardiographic Ndices of Systolic Dysfunction Adjusted for Age Between the KD Patients and the Control Subjects ${ }^{a}$

\begin{tabular}{|c|c|c|c|}
\hline Variables & Control $^{\mathrm{b}}$ & KD Patients $^{\mathrm{c}}$ & P Value \\
\hline Global strain, \% & $20.22 \pm 2.14$ & $17.03 \pm 1.28$ & $<0.001$ \\
\hline Basal global strain, \% & $23.09 \pm 3.89$ & $18.31 \pm 2028$ & \\
\hline Mid-wall global strain,\% & $20.92 \pm 2.36$ & $17.13 \pm 1.67$ & \\
\hline Apical global strain, \% & $16.64 \pm 1.81$ & $15.66 \pm 1.54$ & \\
\hline Global time to peak strain, ms & $289.73 \pm 30.02$ & $293.91 \pm 40.51$ & 0.717 \\
\hline Basal global time to peak strain, $\mathrm{ms}$ & $310.69 \pm 34.75$ & $296.21 \pm 38.33$ & \\
\hline Mid global time to peak strain, $\mathrm{ms}$ & $289.104 \pm 29.03$ & $290.58 \pm 41.60$ & \\
\hline Apical global time to peak strain, ms & $269.39 \pm 31.89$ & $294.93 \pm 54.74$ & \\
\hline Global strain rate, $1 / \mathrm{s}$ & $1.97 \pm 0.25$ & $1.66 \pm 0.16$ & $<0.001$ \\
\hline Basal global strain rate, $1 / \mathrm{s}$ & $2.37 \pm 0.43$ & $1.83 \pm 0.26$ & \\
\hline Mid-wall global strain rate, $1 / \mathrm{s}$ & $1.97 \pm 0.31$ & $1.65 \pm 0.19$ & \\
\hline Apical global strain rate, $1 / \mathrm{s}$ & $1.58 \pm 0.12$ & $1.50 \pm 0.18$ & \\
\hline Global time to peak strain rate, $\mathrm{ms}$ & $188.39 \pm 25.08$ & $179.29 \pm 25.56$ & 0.249 \\
\hline Basal global time to peak strain rate, $\mathrm{ms}$ & $209.57 \pm 34.24$ & $190.51 \pm 36.56$ & \\
\hline Mid global time to peak strain rate, ms & $189.06 \pm 29.15$ & $173.56 \pm 25.66$ & \\
\hline Apical global time to peak strain rate, ms & $166.50 \pm 28.27$ & $173.81 \pm 30.69$ & \\
\hline
\end{tabular}

a Abbreviation: KD, Kawasaki Disease.

$\mathrm{b}(\mathrm{n}=19)$.

C $(\mathrm{n}=32)$

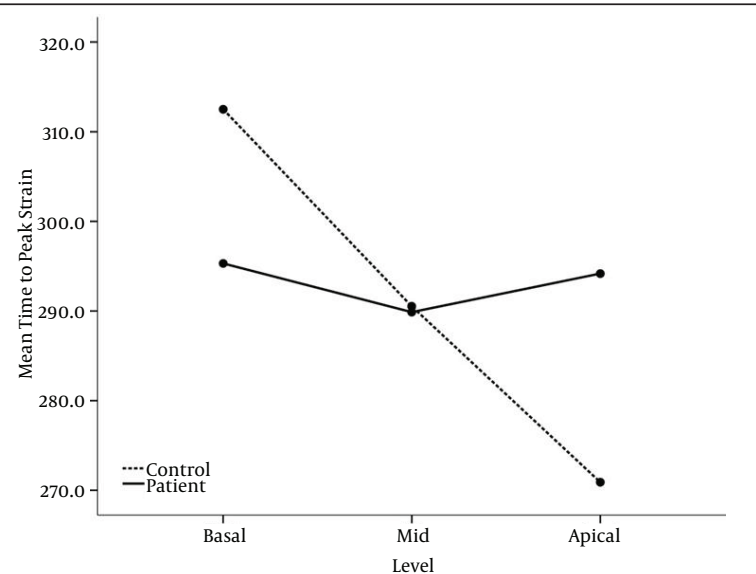

\section{Discussion}

Echocardiographic methods are employed for detecting myocardial dysfunction due to coronary insufficiency in KD patients in early stages $(1,9,10)$. As a noninvasive tool, two-dimensional speckle-tracking echocardiography has been used similarly for both children and adults. This technique describes peak systolic and time to peak systolic S, SR, VP velocity, and longitudinal velocity in 6 views (11-13).

The existing literature contains some information on two-dimensional speckle-tracking indices such as $\mathrm{S}$ and SR for the diagnosis of coronary insufficiency in early stages in KD patients. In this study, we demonstrated that there were reduced $S$ and SR in the KD patients in the four, two, and three-chamber views compared to the controls. We investigated systolic function as a superior marker of coronary insufficiency, although in one study, both diastolic and systolic function indicators were studied (1). Arnold et al. reported systolic SR under exercise as an indicator of myocyte dysfunction in KD patients (1).

In the present study, we observed that global $S$ and SR decreased from basal to apical levels in both KD and normal groups. In line with our findings, Tierney et al. demonstrated that there was impaired relaxation in KD patients in the early phase of the disease and also in advanced KD patients with coronary artery disease during the disease course (14). Yu et al. compared patients in the acute phase of KD with normal controls (10). We also included KD patients in the acute phase of the disease and confirmed that longitudinal basal and mid-wall $S$ in two views were lower in the KD patients, which chimes in with the results of the Yu et al. study (10). Nonetheless, in contrast to our study, the authors of the study in ques-

Figure 2. Comparison of Age Adjusted Changes of Mean Strain (A), Strain Rate (B), Time to Peak Strain (C) and Time to Peak Strain Rate (D) From Basal to Apical Level of Left Ventricular Myocardium Between Kawasaki Patients in Acute Stage and Normal Controls 
tion did not observe significant difference between the control and patient groups regarding systolic SR (10). McCandless et al. used velocity vector imaging to determine $S$ and SR as systolic dysfunction indicators and showed a reduction in $S$ and SR in KD patients in the acute phase compared with a control group, especially in patients who suffered from coronary artery disease (7). McCandless et al. investigated only $S$ and SR as markers of myocardial dysfunction, whereas we studied longitudinal velocity and VP velocity and the time to peak values of these indices, as systolic dysfunction indicators, in addition to $\mathrm{S}$ and SR, and showed that VP velocity was higher in the KD patients in some views. Both VP and long velocity decreased from basal to apical levels in both groups.

The present study has some limitations, first and foremost among which is the unavailability of some echocardiographic techniques, resulting in our inability to perform circumferential measurements. These measurements were studied only during the acute phase of the disease because we could not follow up the patients. We believe that a comparison of the echocardiographic variables of interest in this study between the acute phase and later follow-up may provide more conclusive data. Another limitation in this study is that we could not exclude patients with subclinical myocarditis due to the fact that this condition is undetectable by usual methods and that it requires invasive methods for diagnosis.

Speckle-tracking echocardiography is a new method for the evaluation of systolic ventricular dysfunction. In this study, we used this method in a large sample size and showed that $S$ and SR decreased in the KD patients with different pattern than the controls. However, in contrast to other studies, we demonstrated a drop in $S$ and SR from base to apex in both control and KD groups. Reduced global S and SR may be used as early indicators for systolic ventricular dysfunction in KD patients. Further studies are needed to compare $S$ and SR obtained in the acute phase of KD with that in later follow-up.

\section{Acknowledgements}

We wish to thank the children's parents, who cooperated in this study, and also all the staff of the Pediatric Department for their kind assistance in data gathering.

\section{References}

1. Arnold R, Goebel B, Ulmer HE, Gorenflo M, Poerner TC. An exercise tissue Doppler and strain rate imaging study of diastolic myocardial dysfunction after Kawasaki syndrome in childhood. Cardiol Young. 2007;17(5):478-86.

2. Song ZZ. Abnormal myocardial deformation properties in obese, non-hypertensive children: an ambulatory blood pressure monitoring, standard echocardiographic, and strain rate imaging study. Eur Heart J. 2007;28(13):1662-3.

3. Lorch SM, Sharkey A. Myocardial velocity, strain, and strain rate abnormalities in healthy obese children. J Cardiometab Syndr. 2007;2(1):30-4.

4. Liang H, Zhu M, Feng J, Zhang N, Guo WB. [Evaluation of tissue strain and strain rate imaging for left heart function in children with systemic lupus erythematosus]. Zhonghua Er Ke Za Zhi. 2008;46(11):827-30

5. Dogan V, Ocal B, Orun UA, Ozgur S, Yilmaz O, Keskin M, et al. Strain and strain rate echocardiography findings in children with asymptomatic congenital aortic stenosis. Pediatr Cardiol. 2013;34(5):1152-8.

6. Bussadori C, Moreo A, Di Donato M, De Chiara B, Negura D, Dall'Aglio E, et al. A new 2D-based method for myocardial velocity strain and strain rate quantification in a normal adult and paediatric population: assessment of reference values. Cardiovascular Ultrasound. 2009;7(1):8.

7. McCandless RT, Minich LL, Wilkinson SE, McFadden ML, Tani LY, Menon SC. Myocardial strain and strain rate in Kawasaki disease. Eur Heart J Cardiovasc Imaging. 2013;14(11):1061-8.

8. Sato Y, Tani S, Kunimasa T, Komatsu S, Matsumoto N, Imazeki T, et al. Multidetector-row computed tomography diagnosis of coronary artery aneurysms and collateral vessel after Kawasaki disease in an adult. Cardiovasc Revasc Med. 2006;7(4):243-5.

9. Ge D, Yang XY, Wang RL, Mei L. [Assessment of regional left ventricular systolic function by VSI in children with Kawasaki disease]. Zhongguo Dang Dai Er Ke Za Zhi. 2010;12(4):248-51.

10. Yu JJ, Choi HS, Kim YB, Son JS, Kim YH, Ko JK, et al. Analyses of left ventricular myocardial deformation by speckle-tracking imaging during the acute phase of Kawasaki disease. Pediatr Cardiol. 2010;31(6):807-12.

11. Sadeghpour A, Kyavar M, Madadi S, Ebrahimi L, Khajali Z, Alizadeh Sani Z. Doppler-derived strain and strain rate imaging assessment of right ventricular systolic function in adults late after tetralogy of Fallot repair: an observational study. Anadolu Kardiyoloji Dergisi/The Anatolian Journal of Cardiology. 2013;13:536-42.

12. Konishi K, Dohi K, Tanimura M, Sato Y, Watanabe K, Sugiura E, et al. Quantifying longitudinal right ventricular dysfunction in patients with old myocardial infarction by using speckle-tracking strain echocardiography. Cardiovascular Ultrasound. 2013;11(1):23.

13. Kutty S, Deatsman SL, Nugent ML, Russell D, Frommelt PC. Assessment of regional right ventricular velocities, strain, and displacement in normal children using velocity vector imaging. Echocardiography. 2008;25(3):294-307.

14. Selamet Tierney ES, Newburger JW, Graham D, Baker A, Fulton DR, Colan SD. Diastolic function in children with Kawasaki disease. Int J Cardiol. 2011;148(3):309-12. 